\title{
Prevalence of commonly prescribed medications potentially contributing to urinary symptoms in a cohort of older patients seeking care for incontinence
}

\author{
Mandavi Kashyap ${ }^{1}$, Le Mai Tu² and Cara Tannenbaum ${ }^{1 *}$
}

\begin{abstract}
Background: Several medication classes may contribute to urinary symptoms in older adults. The purpose of this study was to determine the prevalence of use of these medications in a clinical cohort of incontinent patients.

Methods: A cross-sectional study was conducted among 390 new patients aged 60 years and older seeking care for incontinence in specialized outpatient geriatric incontinence clinics in Quebec, Canada. The use of oral estrogens, alpha-blocking agents, benzodiazepines, antidepressants, antipsychotics, ACE inhibitors, loop diuretics, NSAIDs, narcotics and calcium channel blockers was recorded from each patient's medication profile. Lower urinary tract symptoms and the severity of incontinence were measured using standardized questionnaires including the International Consultation on Incontinence Questionnaire. The type of incontinence was determined clinically by a physician specialized in incontinence. Co-morbidities were ascertained by self-report. Logistic regression analyses were used to detect factors associated with medication use, as well as relationships between specific medication classes and the type and severity of urinary symptoms.

Results: The prevalence of medications potentially contributing to lower urinary tract symptoms was $60.5 \%$. Calcium channel blockers (21.8\%), benzodiazepines (17.4\%), other centrally active agents (16.4\%), ACE inhibitors (14.4\%) and estrogens (12.8\%) were most frequently consumed. Only polypharmacy (OR $=4.9,95 \% \mathrm{Cl}=3.1-7.9$ ), was associated with medication use contributing to incontinence in analyses adjusted for age, sex, and multimorbidity. No associations were detected between specific medication classes and the type or severity of urinary symptoms in this cohort.

Conclusion: The prevalence of use of medications potentially causing urinary symptoms is high among incontinent older adults. More research is needed to determine whether de-prescribing these medications results in improved urinary symptoms.
\end{abstract}

Keywords: Older adult, Lower urinary tract symptoms, Incontinence, Medication

\footnotetext{
* Correspondence: cara.tannenbaum@umontreal.ca

'Centre de Recherche, Institut Universitaire de Gériatrie de Montréal, University of Montreal, 4545 Queen Mary Road Suite 4824, Montreal, QC H3W 1W5, Canada

Full list of author information is available at the end of the article
} 


\section{Background}

Lower urinary tract symptoms such as urinary frequency, urgency, nocturia, urinary incontinence and voiding difficulties are highly prevalent and bothersome, impair quality of life and are associated with falls in the elderly [1-4]. The etiology of lower urinary tract symptoms may be singular or multifactorial, with potential contributors encompassing lifestyle habits, underlying bladder or pelvic floor dysfunction, associated co-morbidities and/ or functional factors [5,6]. Many commonly used medications have been proposed to inadvertently interfere with bladder and/or sphincter function, or indirectly impair the ability to toilet [6,7]. The use of oral estrogens, alpha-blocking agents, sedative-hypnotics, antidepressants, antipsychotics, ACE inhibitors, loop diuretics, non-steroidal anti-inflammatory agents (NSAIDS) and calcium channel blockers have all been implicated to some degree in the onset or exacerbation of urinary symptoms [8-23]. Stress urinary incontinence has been associated with the use of alpha-blocking agents, which reduce urethral sphincter tone, and with consumption of ACE inhibitors in patients who experience treatment-related cough $[24,25]$. Loop diuretics promote polyuria and can lead to urinary urgency and frequency, while NSAIDS and other medications known to cause edema may exacerbate nocturia due to nocturnal fluid redistribution. Centrally active medication potentially exert direct effects on the lower urinary tract system by causing relaxation of striated pelvic floor muscles and interfering with afferent sensory messages from the bladder, or even indirectly through their effect on mobility and toileting ability. Calcium channel blockers have been associated with bladder relaxation and impaired emptying, while the mechanism of action of estrogen replacement therapy remains unknown. Acetylcholinesterase inhibitors can precipitate de novo urgency incontinence via enhanced cholinergic activity in the bladder.

Elderly patients who seek care for incontinence have a higher likelihood of multiple morbidity, functional impairment and increased susceptibility to the adverse effects of medications [6]. The prevalence of use of medications potentially contributing to urinary symptoms in older adults seeking care for incontinence remains unknown. A greater understanding of the frequency of use of specific classes of medications among these individuals could help target future research studies and clinical care approaches for reducing medication-risk in patients with urinary symptoms.

The purpose of the present report is to describe the prevalence of use of medications potentially causing or exacerbating lower urinary tract symptoms in a clinical cohort of older men and women presenting to outpatient incontinence clinics for an evaluation of urinary incontinence. Secondary objectives were to determine which factors were associated with the use of these medications, and to investigate whether associations between specific medication classes and the type or severity of urinary symptoms could be detected.

\section{Methods}

\section{Study design, setting and sample}

A cross sectional study of incontinent men and women aged 60 years and older was conducted in Quebec, Canada. Participants were recruited from consecutive new patients presenting to three outpatient urology clinics and three geriatric outpatient incontinence clinics in the Montreal and Sherbrooke areas of Quebec, Canada. The method for recruiting patients has been described previously [26]. Briefly, patients were included in the cohort if they reported a weekly average of one or more episodes of involuntary urine loss during the preceding 3 months. Patients with known dementia (a screening Mini-Mental State Exam score $\leq 24$ ) were excluded from the cohort in order to avoid unreliable self-reported measures. Ten patients with missing data or incomplete medication information were excluded from the analyses. All protocols and procedures were approved by the Institutional Ethics Review Boards of the Institut universitaire de gériatrie de Montréal and the Institut universitaire de gériatrie de Sherbrooke. Informed consent was obtained from each participant prior to data collection.

\section{Data collection and measurement}

A research assistant recorded medication use from bottle labels (or dosette boxes) for prescription drugs and over-the-counter products at the time of the initial clinic visit. Adherence was determined by asking patients if they were currently consuming each medication as prescribed. The name of each prescribed medication, the frequency, daily dose, and treatment duration were recorded. Medications were coded according to the Anatomical Therapeutic Classification [27] for users and non-users of oral estrogens, alpha-blocking agents (antihypertensive and prostate medications), benzodiazepines, antidepressants, antipsychotics, ACE inhibitors, loop diuretics, NSAIDs, lithium, narcotics and calcium channel blockers. Medications known to cause pedal edema such as other GABAergic analgesics (gabapentin, neurontin) and the thiazolinedione hypoglycemic agents were also recorded. Local estrogen formulations were not considered in this analysis.

Storage lower urinary tract symptoms were queried by self-report in accordance with definitions from the International Continence Society [28]. Increased daytime urinary frequency was defined as passing urine 8 or more times per day. Participants reporting the need to wake up at night more than once to urinate were defined as having nocturia. Participants who reported a feeling of urgency and having to rush to the toilet before passing urine (occasionally, most of the time, or all of 
the time) were designated as having urinary urgency. The International Consultation on Incontinence QuestionnaireICIQ-UI-SF was used to determine the presence and severity of urinary incontinence [29]. The severity score for each patient was calculated as the sum of the first 3 items (frequency, amount and impact), ranging from 0 to 21 points. Three severity categories were created: mild (0-5), moderate (6-12), and severe (13-21). The type of incontinence was diagnosed by the treating physician after performing a standard assessment including history, physical examination, stress test, bladder ultrasound for post-void residual urine volume, and urine analysis. A diagnosis of stress, urgency, mixed, urgency with impaired bladder emptying, or functional incontinence was relayed to the research team. Urodynamic tests were not performed unless ordered by the treating physician. Voiding symptoms were not systematically recorded and are not considered in this analysis. Comorbidities and other socio-demographic variables were self-reported by the participant to the research assistant. Comorbidities were based on previous medical diagnoses. Heart disease, stroke and peripheral vascular disease were categorized together as cardiovascular diseases. Musculoskeletal disorders included arthritis, osteoporosis and gout.

\section{Statistical analyses}

Descriptive statistics were used to characterize the participants and to determine the prevalence of use of each medication type. Data were summarized by percentages for categorical variables, and means ( \pm standard deviations) for continuous data. To investigate factors associated with the use of medications contributing to lower urinary tract symptoms, logistic regression analyses were conducted, with medication use inserted in the model as the dependent variable. Age (continuous), sex (binary), individual comorbid conditions (binary), multimorbidity $(\geq 3 \text { conditions })^{29}$ and the presence of polypharmacy $(\geq 5 \text { medications })^{30}$ were regressed against the use of each medication class. Medications belonging to classes presumed to contribute to urinary symptoms were excluded from the polypharmacy variable. To determine whether associations could be detected between the type or severity of lower urinary tract symptoms and the use of drugs from specific medication classes, univariate and multivariate analyses were conducted with the independent variable coded as user versus non user of the targeted medications potentially causing or exacerbating lower urinary tract symptoms (e.g. oral estrogens, alphablocking agents, sedative-hypnotics, antidepressants, antipsychotics, ACE inhibitors, loop diuretics, NSAIDS, calcium channel blockers, and other GABAergic analgesics). The dependent variable was either the presence or absence of each urinary symptom (urgency versus no urgency, nocturia versus nocturia); the type of incontinence (e.g. stress versus other types, urge versus other types); or the severity of incontinence (mild versus moderate or severe). First we regressed "any" medication class as the independent variable, then subgroup analyses were conducted using each distinct medication class separately. Age, sex, polypharmacy and other co-morbidities were included in multivariate analyses if they were independently associated with the outcome at $\mathrm{p}<0.05$. Results are presented as odds ratio (OR) and $95 \%$ confidence intervals (95\% CI). All statistical analyses were conducted using SPSS.

\section{Results}

Three hundred and ninety men and women consented to enroll in the study over a four year time period, representing approximately forty percent of all new patients. The most common reasons for refusing to participate were dementia, institutionalization, disinterest in participating in a research study, and competing acute illness. Data on eligible patients who refused to participate were not available for comparison. Only $1 \%$ of interested individuals met exclusion criteria.

Table 1 shows the characteristics of the participants and the distribution of urinary symptoms and types of incontinence. The mean age was $72.4 \pm 6.4$ years (range 60-90), the mean number of co-morbid conditions was $5.0 \pm 2.5$, and patients consumed on average $6.2 \pm 3.8$ different medications. The most frequent co-morbid conditions included musculoskeletal disorders (self-reported arthritis symptoms), dyslipidemia and depression. All patients were incontinent; the majority (93.1\%) reported moderate to severe symptoms. Forty-five percent of patients were diagnosed with urgency incontinence as the primary diagnosis, explaining the high prevalence of other overactive bladder symptoms such as urinary frequency $(78.7 \%)$, urgency $(91.8 \%)$ and nocturia $(83.6 \%)$ in the cohort.

The prevalence of use of medications potentially contributing to urinary symptoms was $60.5 \%(n=236)$, with a mean of one incriminating drug per patient (Table 2). The top five medication classes were calcium channel blockers (21.8\%), benzodiazepines (17.4\%), other centrally active agents including antipsychotics, antidepressants and narcotics (16.4\%), ACE inhibitors (14.4\%) and oral estrogens (12.8\%). Use of loop diuretics and alphablocking antihypertensive agents was rare.

Polypharmacy was the only factor associated with the use of medications potentially contributing to incontinence in multivariate analysis (Table 3). Individuals consuming 5 or more drugs were almost 5 times more likely to be taking a medication contributing to urinary symptoms, when adjusting for age, sex and comorbidity $(\mathrm{OR}=4.9,95 \% \mathrm{CI}=3.1-7.9)$. Multimorbidity, cardiovascular 
Table 1 Patient characteristics and distribution of urinary symptoms $(n=390)$

\begin{tabular}{|c|c|}
\hline Characteristics & Number (\%) \\
\hline Age in years (Mean $\pm S D$ ) & $72.4 \pm 6.4$ \\
\hline $60-69$ & $150(38.5 \%)$ \\
\hline 70-79 & 175 (44.8\%) \\
\hline $80+$ & $65(16.7 \%)$ \\
\hline Female & $355(91.0 \%)$ \\
\hline Co-morbid conditions ( $\geq 3$ ) & $315(80.7 \%)$ \\
\hline Hypertension & $118(30.3 \%)$ \\
\hline Dyslipidemia & $166(42.6 \%)$ \\
\hline Diabetes & $93(23.8 \%)$ \\
\hline Cardiovascular disease & $133(34.1 \%)$ \\
\hline Depression & $142(36.4 \%)$ \\
\hline Respiratory disease & $65(16.7 \%)$ \\
\hline Renal failure & $20(5.1 \%)$ \\
\hline Musculoskeletal disorders & $385(98.7 \%)$ \\
\hline Polypharmacy ( $\geq 5$ medications) & $244(62.6 \%)$ \\
\hline \multicolumn{2}{|l|}{ Type of incontinence } \\
\hline Urgency & $175(45.0 \%)$ \\
\hline Stress & $56(14.4 \%)$ \\
\hline Mixed & $98(25.0 \%)$ \\
\hline Urgency with impaired emptying & $16(4.1 \%)$ \\
\hline Functional & $45(11.5 \%)$ \\
\hline \multicolumn{2}{|l|}{ Severity of incontinence } \\
\hline Mild & $27(6.9 \%)$ \\
\hline Moderate & $194(49.7 \%)$ \\
\hline Severe & $169(43.4 \%)$ \\
\hline \multicolumn{2}{|l|}{ Other lower urinary tract symptoms } \\
\hline Urinary frequency & $307(78.7 \%)$ \\
\hline Urinary urgency & $358(91.8 \%)$ \\
\hline Nocturia & $326(83.6 \%)$ \\
\hline
\end{tabular}

disease, hypertension, and dyslipidemia were associated with medication use in univariate models but did not remain significant in adjusted models. No associations were found between age or sex and the use of medications potentially contributing to urinary symptoms.

Regression analyses failed to detect significant associations between urinary frequency, urgency or nocturia with any medication class. Nor was any association found between the class of medication and the type or severity of incontinence in univariate or multivariate models adjusted for age, sex, polypharmacy and multiple morbidity (data not shown).

\section{Discussion}

The prevalence of use of medications potentially contributing to urinary symptoms was high (60.5\%) among
Table 2 Prevalence of use of medications potentially contributing to urinary symptoms $(n=390)$

\begin{tabular}{lc}
\hline Medication class & Prevalence $\mathbf{n}(\%)$ \\
\hline Any medication class & $236(60.5 \%)$ \\
Calcium channel blockers & $85(21.8 \%)$ \\
Benzodiazepines & $68(17.4 \%)$ \\
Other centrally active agents* & $65(16.7 \%)$ \\
ACE inhibitors & $56(14.4 \%)$ \\
Oral estrogens & $50(12.8 \%)$ \\
Oral estrogen + progesterone & $12(3.1 \%)$ \\
NSAIDS & $38(9.7 \%)$ \\
GABAergic analgesics & $11(2.8 \%)$ \\
Loop diuretics & $7(1.8 \%)$ \\
Thiazolinedione hypoglycemic agents & $3(0.8 \%)$ \\
Alpha blocking antihypertensives & $1(0.3 \%)$ \\
*Antidepressants, antipsychotics and narcotics were combined.
\end{tabular}

390 new patients presenting for evaluation to a geriatric incontinence clinic. The most common medication classes were calcium channel blockers, benzodiazepines, other centrally active agents, ACE inhibitors and oral estrogens. Loop diuretics and alpha-blocking agents were less frequently prescribed. Patients with polypharmacy were five times more likely to consume potentially deleterious medications, regardless of age, sex and multimorbidity. No specific disease state was associated with medication use when polypharmacy was accounted for. Our analyses were unable to detect significant associations between specific medication classes and the type or severity of lower urinary tract symptoms.

Table 3 Factors associated with the use of medications potentially causing urinary symptoms

\begin{tabular}{lcc}
\hline Predictor & $\begin{array}{c}\text { Crude odds ratio } \\
(\mathbf{9 5 \%} \mathbf{C I})\end{array}$ & $\begin{array}{c}\text { Adjusted odds ratio* } \\
\mathbf{( 9 5 \% ~ C l )}\end{array}$ \\
\hline Age & $1.0(1.0-1.1)$ & $1.0(1.0-1.1)$ \\
Sex & $1.5(0.7-3.0)$ & $1.1(0.5-2.3)$ \\
Polypharmacy $(\geq 5$ drugs $)$ & $5.3(3.4-8.2)$ & $4.9(3.1-7.9)$ \\
Multimorbidity $(\geq 3)$ & $2.0(1.2-3.3)$ & $1.2(0.6-2.03)$ \\
Depression & $1.1(0.7-1.67)$ & $0.7(0.4-1.2)$ \\
Cardiovascular disease & $2.0(1.3-3.0)$ & $1.4(0.8-2.3)$ \\
Hypertension & $2.2(1.4-3.5)$ & $1.5(0.9-2.7)$ \\
Respiratory disease & $1.7(1.0-3.1)$ & $1.4(0.7-2.9)$ \\
Gastrointestinal disorders & $1.5(1.0-2.2)$ & $1.1(0.6-1.9)$ \\
Dyslipidemia & $1.6(1.1-2.4)$ & $0.9(0.5-1.5)$ \\
Renal failure & $1.6(0.6-4.1)$ & $0.7(0.2-2.1)$ \\
Musculoskeletal disorders & $1.3(1.0-1.6)$ & $0.9(0.6-1.2)$ \\
Diabetes & $1.4(0.9-2.3)$ & $0.9(0.5-1.7)$ \\
\hline Adjusted for age, sex, & &
\end{tabular}

${ }^{*}$ Adjusted for age, sex, polypharmacy and multimorbidity. 
The lack of association found in the current study between specific medication classes thought to precipitate symptoms and the type or severity of symptoms mirrors the negative results reported from other large epidemiological studies that examined the relationship between current medication use and urinary incontinence, such as the Boston Area Community Health Survey and the Health, Aging and Body Composition Study [10,21]. One explanation is the cross-sectional nature many of these analyses. Studies that do not account for the timing of administration of a new drug in relation to the appearance of urinary symptoms are limited in their ability to establish causality. Criteria for causality require that the cause precede the effect [30,31]. To meet this criterion, neither incontinence nor the use of a medication class thought to contribute to urinary symptoms should be present at the onset of the study in the population of interest. Observational studies that aim to investigate the effect of specific medication classes on the occurrence and type of urinary symptoms in subgroups of healthy and frail individuals should employ a new-user cohort of individuals without previous medication use or urinary symptoms, rigorous ascertainment of incident incontinence, and sensitive markers of frailty [32]. The strength of the association and the specificity of different treatment-emergent harmful effects in elderly individuals may vary according to whether individuals are healthy, frail or pre-frail, and whether more than one urinary symptom develops. The risk of adverse outcomes may therefore depend on the presence of multiple risk factors and these should be accounted for in the analysis.

Despite this research complexity, a growing body of evidence supports clinicians in their efforts to pursue medication modification as a risk reduction strategy for patients experiencing urinary symptoms. Data exist on post-menopausal hormone therapy examined in the context of two large randomized placebo controlled trials, with urinary symptoms as a post-hoc endpoint $[8,9]$. Findings indicated a two-fold increased risk of de novo stress and mixed incontinence among new users of estrogen replacement therapy [8], a $30 \%$ increase in the onset of urgency incontinence [8] and a significant exacerbation in the amount and frequency of stress and urgency incontinence among women with pre-existing symptoms $[8,9]$. In a case control study, 20/49 women taking alpha-blocking antihyperstensives experienced incontinence compared to $8 / 49$ controls (40.8 vs. $16.3 \%$ ), although the relationship no longer persisted when patients on loop diuretics were excluded from the analysis [12]. Discontinuation of alpha-blocking agents in 18 women with incontinence resulted in 13 (72\%) reporting total or almost complete resolution of their urinary symptoms [12]. Clinical data from a series of 172 elderly hypertensive and heart failure patients found that the use of loop but not thiazide diuretics was significantly associated with increased urinary frequency, a relationship that persisted when the age of the patient and the use of other cardiovascular diseases were accounted for [16]. New use of calcium channels blockers among 38 men aged 47-89 years old was found to significantly exacerbate voiding symptoms, even when adjusting for other medication use and conditions that could contribute to lower urinary tract symptoms [23]. A number of case reports describe cough-induced stress incontinence upon initiation of an ACE inhibitor, which remits upon discontinuation, and one case series reported a $10 \%$ incidence of severe ACE-inhibitor-induced stress incontinence in diabetic post-menopausal women $[17,24,25]$.

The prevalence of use of medications potentially affecting lower urinary tract symptoms may be underestimated in the current study due to selection bias. Patients with dementia, residents of nursing homes, and acutely ill patients did not participate, and may reasonably have a higher risk of taking acetylcholinesterase inhibitors or consuming multiple medications. Furthermore, the cohort comprised a select sample of patients who were referred to a specialist clinic, and only $40 \%$ of eligible patients consented to provide data. Women were overrepresented. Many patients are referred to subspecialty clinics only when their symptoms are refractory to firstline management, so medications contributing to urinary symptoms already may have been discontinued. Patients successfully treated by a primary care provider by stopping a medication (e.g., a diuretic), would not be referred to the incontinence clinic and would not be included in the current sample. Multimorbidity was not a significant predictor when polypharmacy was accounted for, and this could partially be explained by collinearity between the two variables, or by virtue of polypharmacy acting as a proxy of disease severity. Although an attempt was made to investigate the association between medication usage and the type of incontinence, the etiology of incontinence is often multifactorial in older persons, so misclassification or lack of specificity of the type of incontinence may have contributed to the null effect of these analyses.

\section{Conclusion}

The prevalence of use of medications potentially causing or exacerbating lower urinary tract symptoms was high $(60.5 \%)$ in this clinical cohort of new patients presenting for a geriatric evaluation of urinary incontinence. More research is needed to determine whether de-prescribing these medications results in improved urinary symptoms.

\section{Competing interests}

The authors have no financial conflicts of interest to disclose with respect to this work. Cara Tannenbaum declares having received honoraria from 
Pfizer, Allergen and Ferring Pharmaceuticals during the past three years. Le Mai Tu declares having consulted for Pfizer, Astellas, Watson, Medtronic and Allergen. Although both Cara Tannenbaum and Le Mai Tu have served as consultants or speakers at symposium for these companies related to the pharmacologic treatment of incontinence in the elderly with bladder relaxant medication, the authors do not feel that they were influenced in any way in the reporting of the results from the current clinical cohort study (which was not funded by industry).

\section{Authors' contributions}

Study concept and design: CT and MK; Acquisition of subjects and/or data: CT and LMT; Analysis and interpretation of data: MK and CT; Preparation of manuscript: MK, CT and LMT. All authors read and approved the final manuscript.

\section{Sponsor's role}

This study was funded by an operating grant from the Canadian Institutes of Health Research. The sponsor had no role in the design, methods, subject recruitment, data collection, analysis and preparation of the paper.

\section{Funding sources}

The research reported in this manuscript was supported by an operating grant from the Canadian Institutes of Health Research to Cara Tannenbaum. There were no other sources of funding. The funding sponsor had no role in the design, conduct, analysis, interpretation of the results from this study, nor in the drafting or decision to submit this article for publication.

\section{Meeting attended and presented}

American Geriatric Society conference 2012 Seattle, WA, USA.

\section{Author details}

${ }^{1}$ Centre de Recherche, Institut Universitaire de Gériatrie de Montréal, University of Montreal, 4545 Queen Mary Road Suite 4824, Montreal, QC H3W 1W5, Canada. Institut Universitaire de Gériatrie de Sherbrooke, Université de Sherbrooke, Sherbrooke, QC, Canada.

Received: 4 October 2012 Accepted: 3 June 2013

Published: 10 June 2013

\section{References}

1. Chiarelli PE, Mackenzie LA, Osmotherly PG: Urinary incontinence is associated with an increase in falls: a systematic review. Aust $J$ Physiother 2009, 55(2):89-95.

2. Foley AL, Loharuka S, Barrett JA, Mathews R, Williams K, McGrother CW, Roe BH: Association between the Geriatric Giants of urinary incontinence and falls in older people using data from the Leicestershire MRC Incontinence Study. Age Ageing 2012, 41(1):35-40.

3. Sexton CC, Coyne KS, Thompson C, Bavendam T, Chen Cl, Markland A: Prevalence and effect on health-related quality of life of overactive bladder in older Americans: Results from the Epidemiology of Lower Urinary Tract Symptoms Study. J Am Geriatr Soc 2011, 59:1465-1470.

4. Irwin DE, Milsom I, Hunskaar S, Reilly K, Kopp Z, Herschorn S, Coyne K, Kelleher C, Hampel C, Artibani W, Abrams P: Population-based survey of urinary incontinence, overactive bladder, and other lower urinary tract symptoms in five countries: Results of the EPIC study. Eur Urol 2006, 50:1306-1314.

5. Tannenbaum C, Gray M, Hoffstetter S, Cardozo L: Comorbidities associated with bladder dysfunction. Int I Clin Pract 2013, 67:105-113.

6. DuBeau CE, Kuchel GA, Johnson T 2nd, Palmer MH, Wagg A: Fourth International Consultation on Incontinence. Incontinence in the frail elderly: report from the 4th International Consultation on Incontinence. Neurourol Urodyn 2010, 29(1):165-178.

7. Tsakiris $P$, Oelke M, Michel MC: Drug-induced urinary incontinence. Drugs Aging 2008, 25:541-549.

8. Hendrix SL, Cochrane BB, Nygaard IE, Handa VL, Barnabei VM, Iglesia C, Aragaki A, Naughton MJ, Wallace RB, McNeeley SG: Effects of estrogen with and without progestin on urinary incontinence. JAMA 2005, 293:935-948.

9. Grady D, Brown JS, Vittinghoff E, Applegate W, Varner E, Snyder T, HERS Research Group: Postmenopausal hormones and incontinence: the Heart and Estrogen/Progestin Replacement Study. Obstet Gynecol 2001, 97:116-120.

10. Ruby CM, Hanlon JT, Boudreau RM, Newman AB, Simonsick EM, Shorr RI, Bauer DC, Resnick NM: Health, Aging and Body Composition Study: The effect of medication use on urinary incontinence in communitydwelling elderly women. J Am Geriatr Soc 2010, 58:1715-1720.

11. Wuerstle MC, Van Den Eeden SK, Poon KT, Quinn VP, Hollingsworth JM, Loo RK, Jacobsen SJ, Urologic Diseases in America Project: Contribution of common medications to lower urinary tract symptoms in men. Arch Intern Med 2011, 171:1680-1682.

12. Marshall HJ, Beevers DG: Alpha-adrenoceptor blocking drugs and female urinary incontinence: prevalence and reversibility. Br J Clin Pharmacol 1996, 42:507-509.

13. Finkelstein $\mathrm{MM}$ : Medical conditions, medications, and urinary incontinence: analysis of a population-based survey. Can Fam Phys 2002. 48:96-101.

14. Landi F, Cesari M, Russo A, Onder G, Sgadari A, Bernabei R, Silvernet-HC Study Group: Benzodiazepines and the risk of urinary incontinence in frail older persons living in the community. Clin Pharmacol Ther 2002, 72:729-734.

15. Ruby CM, Hanlon JT, Fillenbaum GG, Pieper CF, Branch LG, Bump RC: Medication use and control of urination among community-dwelling older adults. J Aging Health 2005, 17:661-674.

16. Ekundayo OJ, Markland A, Lefante C, Sui X, Goode PS, Allman RM, Ali M, Wahle C, Thornton PL, Ahmed A: Association of diuretic use and overactive bladder syndrome in older adults: a propensity score analysis. Arch Gerontol Geriatr 2009, 49:64-68.

17. Lee YJ, Chiang YF, Tsai JC: Severe nonproductive cough and coughinduced stress urinary incontinence in diabetic postmenopausal women treated with ACE inhibitor. Diabetes Care 2000, 23:427-428.

18. Meigs JB, Barry MJ, Giovannucci E, Rimm EB, Stampfer MJ, Kawachi I: Incidence rates and risk factors for acute urinary retention: the health professionals follow up study. J Urol 1999, 162:376-382.

19. Gates MA, Hall SA, Chiu GR, Kupelian V, Fitzgerald MP, Link CL, McKinlay JB: Nonsteroidal antiinflammatory drug use and lower urinary tract symptoms: results from the Boston area community health survey. Am J Epidemiol 2011, 173:1022-1031.

20. Hall SA, Chiu GR, Kaufman DW, Wittert GA, Link CL, McKinlay JB: Commonly used antihypertensives and lower urinary tract symptoms: results from the Boston Area Community Health (BACH) Survey. BJU Int 2012, 109(11):1676-1684.

21. Hall SA, Yang M, Gates MA, Steers WD, Tennstedt SL, McKinlay JB: Associations of Commonly Used Medications with Urinary Incontinence in a Community Based Sample. J Urol 2012, 188(1):183-189.

22. Hall SA, Maserejian NN, Link CL, Steers WD, McKinlay JB: Are commonly used psychoactive medications associated with lower urinary tract symptoms? Eur J Clin Pharmacol 2012, 68:783-791.

23. Hughes JD, Coles MA, Joyce A: Calcium channel blocker associated lower urinary tract symptoms in males: an Australian retrospective observational study. Qual Prim Care 2011, 19:223-231.

24. Menefee SA, Chesson R, Wall LL: Stress urinary incontinence due to prescription medications: alpha-blockers and angiotensin converting enzyme inhibitors. Obstet Gynecol 1998, 91:853-854.

25. Casanova JE: Incontinence after use of enalapril. J Urol 1990, 143:1237-1238.

26. Tannenbaum C, Brouillette J, Korner-Bitensky N, Dumoulin C, Corcos J, le Tu M, Lemieux MC, Ouellet S, Valiquette L: Creation and testing of the Geriatric Self-Efficacy Index for Urinary Incontinence. J Am Geriatr Soc 2008, 56(3):542-547.

27. WHO Collaborating Centre for Drug Statistics Methodology: Anatomical Therapeutic Chemical (ATC) classification index with Defined Daily Dose. Geneva: WHO; 2003

28. Abrams $P$, Cardozo L, Fall $M$, Griffiths $D$, Rosier $P$, Ulmsten $U$, van Kerrebroeck $P$, Victor A, Wein A, Standardisation Sub-committee of the International Continence Society: The standardization of terminology of lower urinary tract function: report from the Standardisation Sub-committee of the International Continence Society. Neurourol Urodyn 2002, 21:167-178.

29. Avery K, Donovan J, Peters TJ, Shaw C, Gotoh M, Abrams P: ICIQ: a brief and robust measure for evaluating the symptoms and impact of urinary incontinence. Neurourol Urodyn 2004, 23:322-330. 
30. Kramer MS, Leventhal JM, Hutchinson TA, Feinstein AR: An algorithm for the operational assessment of adverse drug reactions. I. Background, description, and instructions for use. JAMA 1979, 242(7):623-632.

31. Naranjo CA, Busto U, Sellers EM, et al: A method for estimating the probability of adverse drug reactions. Clin Pharmacol Ther 1981, 30(2):239-245.

32. Avorn J: In defense of pharmacoepidemiology - embracing the Yin and Yang of drug research. N Engl J Med 2007, 357:2219-2221.

doi:10.1186/1471-2318-13-57

Cite this article as: Kashyap et al:: Prevalence of commonly prescribed medications potentially contributing to urinary symptoms in a cohort of older patients seeking care for incontinence. $B M C$ Geriatrics 2013 13:57.

\section{Submit your next manuscript to BioMed Central and take full advantage of:}

- Convenient online submission

- Thorough peer review

- No space constraints or color figure charges

- Immediate publication on acceptance

- Inclusion in PubMed, CAS, Scopus and Google Scholar

- Research which is freely available for redistribution 